\title{
Insomnia, Sleep Apnea, and Circadian Misalignment as a "Three-arm" Contributor to Anxiety and Depression During Pregnancy
}

\author{
Miguel Meira e Cruz ${ }^{1,2,3}$ (D) Alexander Sweetman ${ }^{4}$
}

Received: 13 May 2021 / Revised: 5 August 2021 / Accepted: 12 August 2021 / Published online: 16 August 2021

(c) The Author(s), under exclusive licence to Springer Nature Singapore Pte Ltd. 2021

Anxiety and depression symptoms often occur during pregnancy. The overlap between symptoms related with pregnancy itself, and concomitant affective disorders can significantly affect the mother's health, potentially impacting delivery associated outcomes. Controversial issues persist regarding the relationship between sleep disturbance and psychosomatic outcomes during pregnancy. Of interest, a recent study by Lin and colleagues reported a strong association between sleep disturbance, anxiety, and depressive symptoms among pregnant women during the COVID-19 pandemic [1]. Although cross-sectional in nature, the main observations of this study raise important implications regarding the development of sleep disturbances during pregnancy, which may act as an explanatory mechanism for increased risk of psychosocial stress. Importantly, the authors highlighted the potential role of clinical sleep disorders including insomnia, and circadian misalignment on mental and physical health. It is also important to consider the role of obstructive sleep apnea (OSA), as a risk factor for reduced mental health during pregnancy, and the overlap of OSA with other sleep disorders.

Notwithstanding, these results conflict with those of Zou et al. [2] showing that during COVID-19 epidemic in China pregnant women had lower rates of depression (5.3\% prevalence), anxiety (6.8\%), and insomnia (2.6\%), compared to

Miguel Meira e Cruz

mcruz@medicina.ulisboa.pt

1 Sleep Unit, Centro Cardiovascular da Universidade de Lisboa, Lisbon School of Medicine, Av. Prof Egas Moniz, 1649-028 Lisboa, Portugal

2 International Center on Clinical Sleep Medicine and Research, Escola Bahiana de Medicina e Saúde Pública, Salvador, Brazil

3 Neuroimmune Pain Interface Lab, Faculdade São Leopoldo Mandic, Campinas, Brazil

4 Adelaide Institute for Sleep Health, FHMRI Sleep Health, College of Medicine and Public Health, Flinders University, Bedford Park, Australia estimates reported by Li and colleagues [1,2]. Different reasons could be implicated in these contrasting findings such as differences in the study population, assessment tools and criteria (e.g., a different threshold on the PHQ-9 depression measure), and the temporal impact of COVID-19 epidemic during data collection. Despite these contrasting prevalence estimates, individual characteristics both associated to sleep disturbance (i.e., insomnia) [3] and to circadian clock phenotypes likely play an important role in the link between disturbed sleep and psycho-emotional health during pregnancy $[1,4]$.

Difficulties initiating sleep (one of the three core nocturnal symptoms of insomnia disorder) were strongly associated with the presence of both anxiety and depression symptoms. Although this study did not assess for difficulties maintaining sleep or early morning awakenings (the other main nocturnal insomnia symptoms), or chronicity of insomnia symptoms, previous research has established insomnia as an important risk factor associated with the development of depression [5] and has reported a high prevalence of insomnia symptoms during pregnancy [6]. Anxiety and depression are also powerful psychological mechanisms that may impact sleep physiology. Indeed, relationships between circadian rhythmicity, sleep and mood have been established in gestational insomnia, that may lead to post-partum depression [7]. The most effective treatment for insomnia, Cognitive Behavioural Therapy for insomnia (CBTi), improves sleep and symptoms of depression, anxiety and stress [8]. A recent randomized controlled trial also recently reported that (digital) CBTi improves sleep during and following pregnancy, compared to sleep education control [9]. As highlighted by Lin and colleagues [1], targeted interventions to improve sleep conditions among pregnant women may be important in improving associated mental health symptoms.

Importantly, insomnia and OSA commonly co-occur [10]. OSA is characterized by frequent narrowing (hypopnea) and/or closure (apnea) of the upper airway during sleep, resulting in reduced oxygen saturation, cortical 
arousals, sleep fragmentation, and daytime sleepiness/ fatigue [11]. Like insomnia, OSA is also associated with symptoms of anxiety and depression $[10,11]$. Co-morbid insomnia and sleep apnea (COMISA) may be associated with increased cardiovascular disease risk $[12,13]$ and worse mental health and quality of life [13, 14]. Although pregnancy may be associated with increased prevalence of both insomnia and OSA [15, 16], few studies have investigated the co-occurrence of insomnia and OSA during pregnancy, or the effect of COMISA on depression, anxiety and stress during or following pregnancy.

Gestational sleep disordered breathing (SDB) often affects pregnant women, ranging from simple snoring to complete cessation of breathing with recurrent airflow interruptions usually accompanied by oxygen desaturation and fragmented sleep (i.e., OSA). SDB may also present as snoring and/or self-reported 'poor sleep quality').

Pregnancy is a particularly vulnerable moment of life where sleep and psychological health often interact, and may perpetuate multiple psychological (e.g., anxiety, stress, depression), and physical consequences (e.g., disturbed metabolism, immunity suppression). These intriguing interactions may also be influenced by a significant increase in body mass index and cardiorespiratory changes due to altered circuitry of several regulatory processes. For instance, neurohumoral changes including those related to central modulation of leptin and ghrelin may increase the likelihood of overweight and obesity, as well as mediate sympathetic activation and cardiac function [16]. These changes may favour increased symptoms of fatigue and exhaustion commonly reported during pregnancy. At the same time hormonal modifications may affect sleep quality and sleep duration. Interestingly, excessive daytime sleepiness and total sleep duration both increase during pregnancy, potentially due to either the deterioration in subjective sleep quality or hormonal changes.

This may also result from neuroendocrine functional pathways such as those implicated in the lower progesterone levels. Estrogen-mediated nasopharyngeal edema and rhinitis are common risk factors for gestational SDB. There are also some factors associated with pregnancy that may be protective against OSA. For example, the proneness for a lateral sleep position (in which airway closure is less likely to occur), a reduction in the percentage of rapid-eye-movement sleep (in which airway closure events are more common than in deep sleep), and the rightward shift of the oxyhemoglobin dissociation curve (promoting placental oxygen delivery) may serve as protective factors against exacerbation of gestational sleep apnea [16]. BMI may be indicated as a significant predictor of gestational SDB, accounting for an increase in sleep apnea prevalence from $10.5 \%$ in the first trimester to $26.7 \%$ in the third trimester [15].
Furthermore, circadian misalignment (i.e., a mismatch between the internal body clock and the environmental light-dark cycle) and chronotype (e.g., an individual's inclination to sleep at a specific time) may affect the circadian timing system and interactions with sleep, psychological, cardiovascular and metabolic outcomes. For example, the evening chronotype is more likely to be associated with sleep onset insomnia, anxiety, and depression in adults and paediatric samples $[18,19]$. Interestingly, insomnia, OSA, and circadian misalignment may frequently co-occur in a more complex condition. Among pregnant women, all three factors potentially interact and impair homeostatic stability, either related to sleep or to neurohormonal milieu underpinning daytime function and mood.

These important findings have promoted awareness of the link between poor sleep and psychosocial stress during pregnancy [1]. Future research should also explore the interactive role of insomnia, OSA, and circadian misalignment on mental and physical health during pregnancy. Developing a greater understanding of the interactions between these sleep disorders/symptoms may guide more effective treatment approaches that also prevent and improve mental health problems during and following pregnancy.

Funding The authors declare that they don't have any financial arrangements or commercial connections.

\section{Declarations}

Conflict of interest The authors declare to have no conflict of interest.

\section{References}

1. Lin W, Wu B, Chen B, Lai G, Huang S, Li S, Wang Y. Sleep conditions associate with anxiety and depression symptoms among pregnant women during the epidemic of COVID-19 in Shenzhen. J Affect Disord. 2021;281:567-73.

2. Zhou Y, Shi H, Liu Z, et al. The prevalence of psychiatric symptoms of pregnant and non-pregnant women during the COVID-19 epidemic. Transl Psychiatry. 2020;10:319. https://doi.org/10.1038/ s41398-020-01006-x.

3. Cuamatzi-Castelan AS, Kalmbach DA, Atkinson R, Sangha R, Bazan L, O'Brien LM, Drake CL. Insomnia in late pregnancy: characterizing phenotypes and identifying associated factors. Sleep. 2019;42:138.

4. Obeysekare JL, Cohen ZL, Coles ME, Pearlstein TB, Monzon C, Coles E, Sharkey K. Delayed sleep timing and circadian rhythms in pregnancy and transdiagnostic symptoms associated with postpartum depression. Transl Psychiatry. 2011;10:14.

5. Baglioni C, Battagliese G, Feige B, Spiegelhalder K, Nissen C, Voderholzer U, Riemann D. Insomnia as a predictor of depression: a meta-analytic evaluation of longitudinal epidemiological studies. J Affect Disord. 2011;135(1-3):10-9. 
6. Sedov ID, Anderson NJ, Dhillon AK, Tomfohr-Madsen LM. Insomnia symptoms during pregnancy: a meta-analysis. J Sleep Res. 2021;30(1):13207.

7. Gallaher KGH, Slyepchenko A, Frey BN, Urstad K, Dørheim SK. The role of circadian rhythms in postpartum sleep and mood. Sleep Med Clin. 2018;13(3):359-74.

8. Sweetman A, Lovato N, Micic G, Scott H, Bickley K, Haycock J, Lack L. Do symptoms of depression, anxiety or stress impair the effectiveness of cognitive behavioural therapy for insomnia? A chart-review of 455 patients with chronic insomnia. Sleep Med. 2020;75:401-10.

9. Kalmbach DA, Cheng P, O'Brien LM, Swanson LM, Sangha R, Sen S, Drake CL. A randomized controlled trial of digital cognitive behavioral therapy for insomnia in pregnant women. Sleep Med. 2020;72:82-92.

10. Sweetman AM, Lack LC, Catcheside PG, Antic NA, Chai-Coetzer CL, Smith SS, Douglas JA, McEvoy RD. Developing a successful treatment for co-morbid insomnia and sleep apnoea. Sleep Med Rev. 2017;33:28-38. https://doi.org/10.1016/j.smrv.2016.04.004.

11. Santos I, de Sousa Rocha I, Gozal D, e Cruz MM. Obstructive sleep apnea, shift work and cardiometabolic risk. Sleep Med. 2020. https://doi.org/10.1016/j.sleep.2020.05.013.

12. Meira E, Cruz M, Salles C, Gozal D. A reappraisal on the associations between sleep-disordered breathing, insomnia and cardiometabolic risk. Am J Respir Crit Care Med. 2021. https://doi.org/ 10.1164/rccm.202102-0337LE.

13. Meira E Cruz M, Kryger MH, Morin CM, Palombini L, Salles C, Gozal D. Comorbid Insomnia and Sleep Apnea: mechanisms and implications of an underrecognized and misinterpreted sleep disorder. Sleep Med. 2021;84:283-288. https://doi.org/10.1016/j. sleep.2021.05.043.

14. Jeon B, Luyster FS, Callan JA, Chasens ER. Depressive symptoms in comorbid obstructive sleep apnea and insomnia: an integrative review. Western J Nurs Res. 2021;3:0193945921989656.

15. Pien GW, Pack AI, Jackson N, Maislin G, Macones GA, Schwab RJ. Risk factors for sleep-disordered breathing in pregnancy. Thorax. 2014;69(4):371-7. https://doi.org/10.1136/thora xjnl-2012-202718.

16. Silvestri R, Aricò I. Sleep disorders in pregnancy. Sleep Sci. 2019;12(3):232-9. https://doi.org/10.5935/1984-0063.20190098.

17. Harris M, Glozier N, Ratnavadivel R, Grunstein RR. Obstructive sleep apnea and depression. Sleep Med Rev. 2009;13(6):437-44.

18. Eid B, Bou Saleh M, Melki I, Torbey PH, Najem J, Saber M, El Osta N, Rabbaa KL. Evaluation of chronotype among children and associations with bmi, sleep, anxiety, and depression. Front Neurol. 2020;5(11):416. https://doi.org/10.3389/fneur.2020.00416 (PMID:32581995;PMCID:PMC7291378).

19. Lucassen EA, Zhao X, Rother KI, Mattingly MS, Courville AB, Csako G, Cizza G. Sleep Extension Study Group Evening chronotype is associated with changes in eating behavior, more sleep apnea and increased stress hormones in short sleeping obese individuals. PLoS ONE. 2013;8(3):e56519.

Publisher's Note Springer Nature remains neutral with regard to jurisdictional claims in published maps and institutional affiliations. 